\title{
MATHEMATICAL ANALYSIS OF CONTINUOUS TIME ACTIVE AND ADAPTIVE DYNAMICS OF ARTIFICIAL NEURAL NETWORK IN STAR SHAPE
}

\author{
MILOŠ KřIVAN
}

\begin{abstract}
The present paper gives a detailed mathematical description of continuous time active and adaptive dynamics of an artificial neural network, based on adaptive resonance theory and consisting in solving systems of differential equations. The mathematical description uses the example of a simple star-shaped artificial neural network for two differently parameterized cases of general equations including the evaluation of both learning methods and both their functions.
\end{abstract}

\section{INTRODUCTION}

Adaptive Resonance Theory (ART) describes the process of elaboration (active dynamics) and storage (adaptive dynamics) information mediated by sensory perception in the human brain. ART considers two progressions, active and adaptive dynamics, that is, discrete time (ART1) and continuous time (ART2), respectively. The first progression is modeled by difference (algebraic) equations while the second one by differential equations.

ART $[4,5]$ neural network is composed of two layers of neurons fully mutually connected. The processing of the information on the mutual oscillations of states of both layers continues until stable state is found. This process is called resonance.

ART1 [1] neural network quickly processes and easily stores the information in discrete portions so that it is applicable as an effective tool for solving many practical problems of various fields of human activity.

ART2 $[2,3]$ neural network processes and stores continuously changing information presented for a given period so that it more realistic models biological processes in the information systems of living organisms. The storing of information in a long-term memory of second type network can be done, for example, in two different ways. The first one is termed the "instar" learning and the second one the "outstar" learning. Both methods prefigure qualitatively different functions of the learned instar and outstar neural network respectively.

$M S C$ (2010): primary 91A28, 91A80; secondary 62-00.

Keywords: artificial neural network, adaptive resonance theory, network dynamics, learning methods, conditioned reflex, Pavlov's experiment, associative memory. 


\section{Definition of terms}

We define an artificial neural network as a directed graph with vertices and edges dynamically evaluated, i.e., as the ordered quintuple $[V, E, \varepsilon, y, w]$ :

$V$ set of vertices (neurons),

$E$ set of edges (synapses),

$\varepsilon \quad$ mapping of incidence of edges with vertices $(\varepsilon: E \rightarrow V \times V)$,

$y$ dynamic evaluation of vertices $(y: V \times \boldsymbol{t} \rightarrow R)$,

$w$ dynamic evaluation of edges $(w: \varepsilon(E) \times \boldsymbol{T} \rightarrow R)$

where $\forall t \in \boldsymbol{t}$ or $\forall T \in \boldsymbol{T}$ we put $y([i, t]) \equiv y_{i}(t)$ and $w([i, j, T]) \equiv w_{i j}(T)$.

The vector $\vec{y}(t)=\left[y_{i}(t) \mid i \in V\right]$ is called the network state at time $t$ and the vector $\vec{w}(T)=\left[w_{i j}(T) \mid[i, j] \in V \times V\right]$ is called the network configuration at time $T$ $\left(\forall[i, j] \notin \varepsilon(E) \Rightarrow w_{i j}(T)=0\right)$. The state or configuration of a network as a vector function of time $t$ or $T$ is termed active dynamics or adaptive dynamics of a neural network, respectively.

Using time separation of the active and adaptive dynamics, we expressed the fact that a neural network works in two time-independent active and adaptive modes.

The active or adaptive dynamics of a neural network in continuous time can be defined as a solution vector of the following systems of differential equations [4]:

$$
\frac{\mathrm{d}}{\mathrm{d} t} x_{j}(t)+x_{j}(t)=\sum_{i} f_{i}\left(x_{i}(t-\Delta t)\right) w_{i j}-\vartheta_{j}
$$

or

$$
\frac{\mathrm{d}}{\mathrm{d} T} w_{i j}(T)+\beta g_{j}\left(x_{j}(T)\right) w_{i j}(T)=\alpha f_{i}\left(x_{i}(T)\right) g_{j}\left(x_{j}(T)\right)
$$

where $i, j \in V, \alpha, \beta \in[0,1]$. Here, analogously to biological neural network, we call

$x_{i} \quad$ potential of the $i$-th neuron,

$f_{i} \quad$ activation function of the $i$-th neuron $\left(f_{i}\left(x_{i}\right)=y_{i}\right)$,

$g_{j} \quad$ adaptation function of the $j$-th neuron,

$\vartheta_{j} \quad$ threshold of the $j$-th neuron,

$w_{i j}$ synaptic weight links of the $i$-th neuron to the $j$-th neuron,

$\alpha \quad$ measure of plasticity of synapses,

$\beta \quad$ measure of elasticity of synapses,

$\Delta t$ signal delay period.

In the usual formulation of the potential and activation functions

$$
x_{j}=\sum_{i} y_{i} w_{i j}, \quad f(x)=\frac{1}{1+\mathrm{e}^{-p(x-\vartheta)}},
$$

we perceive the parameter $\vartheta$ as the threshold of a neuron. Its under-flowing or overflowing by the potential of a neuron causes the inhibition or excitation of a neuron, respectively. In our formulation of the potential and activation functions

$$
x_{j}=\sum_{i} y_{i} w_{i j}-\vartheta_{j}, \quad f(x)=\frac{1}{1+\mathrm{e}^{-p x}},
$$


we perceive the parameter $-\vartheta$ as the external stimulus activating a network by injection directly into neuron, on its value the potential of a neuron is initiated $x_{j}(0)=-\vartheta_{j}$. Then the vector $\vec{x}(0)=\left[x_{j}(0) \mid j \in V\right]$ means the network input and the network state means the network output. The network function which sets the network output using a network input (during active dynamics) is defined as the vector function

$$
\vec{F}(\vec{x}(0))=\vec{y}(\infty)
$$

and the network configuration is then a network function parameter.

\section{INSTAR NETWORK}

Let us have the neural network (see Figure 1) where for simplicity: let us consider the activation and adaptation function as identity and let us set the measure of plasticity consistently with the measure of elasticity equal to one. Then, assuming zero initial conditions for $-\vartheta_{j}=I_{j}>0$ where $j \in\{0,1,2,3\}$, we obtain from $(2.1)$ and (2.2) next active and adaptive dynamics.

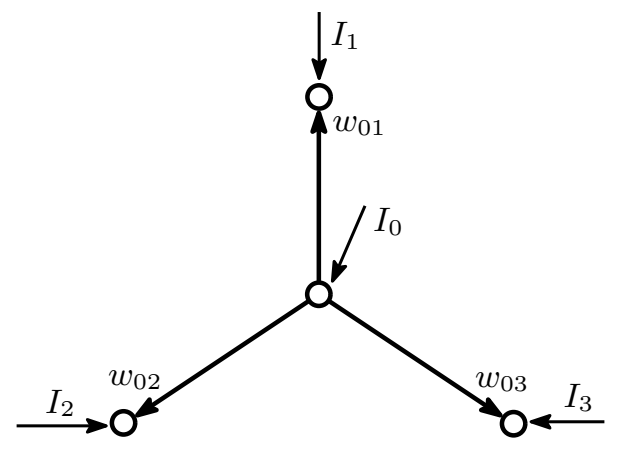

Figure 1. Instar network.

Active dynamic:

$$
\frac{\mathrm{d}}{\mathrm{d} t} x_{0}(t)+x_{0}(t)=I_{0}
$$

i.e.,

$$
x_{0}(t)=I_{0}\left(1-\mathrm{e}^{-t}\right)
$$

i.e.,

$$
\lim _{t \rightarrow \infty} x_{0}(t)=x_{0}(\infty)=I_{0}
$$

and for $j \in\{1,2,3\}$

$$
\frac{\mathrm{d}}{\mathrm{d} t} x_{j}(t)+x_{j}(t)=x_{0}(t-\Delta t) w_{0 j}+I_{j},
$$

i.e.,

$$
x_{j}(t)=I_{0} w_{0 j}\left(1-q(t) \mathrm{e}^{-t}\right)+I_{j}\left(1-\mathrm{e}^{-t}\right), \quad q(t)=\left(1+t \mathrm{e}^{\Delta t}\right),
$$


i.e.,

$$
\lim _{t \rightarrow \infty} x_{j}(t)=x_{j}(\infty)=I_{0} w_{0 j}+I_{j} .
$$

Adaptive dynamics:

i.e.,

$$
\frac{\mathrm{d}}{\mathrm{d} T} w_{0 j}(T)+x_{j}(\infty) w_{0 j}(T)=x_{0}(\infty) x_{j}(\infty),
$$

$$
\frac{\mathrm{d}}{\mathrm{d} T} w_{0 j}(T)=-\left(a w_{0 j}^{2}(T)-b w_{0 j}(T)+c\right)
$$

for $j \in\{1,2,3\}$, where

$$
a=I_{0}, \quad b=I_{0}^{2}-I_{j}, \quad c=-I_{0} I_{j},
$$

i.e., after separating the variables,

$$
T=-\int \frac{1}{a w_{0 j}^{2}-b w_{0 j}+c} \mathrm{~d} w_{0 j}
$$

i.e., generally,

$$
T=-\frac{1}{\sqrt{D}} \ln \left|\frac{w_{0 j}-\frac{b+\sqrt{D}}{2 a}}{w_{0 j}-\frac{b-\sqrt{D}}{2 a}}\right|+C, \quad D=b^{2}-4 a c=\left(I_{0}^{2}+I_{j}\right)^{2}>0
$$

where $b-\sqrt{D}<0<b+\sqrt{D}$ and $C$ is an integration constant.

Firstly, let us consider $w_{0 j} \notin\left[\frac{b-\sqrt{D}}{2 a}, \frac{b+\sqrt{D}}{2 a}\right]$. Then, we get the hyperbolic behavior (see Figure 2)

i.e., for $T \neq C$,

$$
\mathrm{e}^{-\sqrt{D}(T-C)}=\frac{w_{0 j}-\frac{b+\sqrt{D}}{2 a}}{w_{0 j}-\frac{b-\sqrt{D}}{2 a}}
$$

$$
w_{0 j}(T)=\frac{1}{2 a}\left(b-\sqrt{D} \frac{\mathrm{e}^{-\sqrt{D}(T-C)}+1}{\mathrm{e}^{-\sqrt{D}(T-C)}-1}\right)
$$

i.e.,

$$
\lim _{T \rightarrow \infty} w_{0 j}(T)=w_{0 j}(\infty)=\frac{b+\sqrt{D}}{2 a}=I_{0}
$$

and

$$
\lim _{T \rightarrow-\infty} w_{0 j}(T)=w_{0 j}(-\infty)=\frac{b-\sqrt{D}}{2 a}=-\frac{I_{j}}{I_{0}} .
$$

For $T=0$, we get

$$
C=\frac{1}{\sqrt{D}} \ln \frac{w_{0 j}(0)-\frac{b+\sqrt{D}}{2 a}}{w_{0 j}(0)-\frac{b-\sqrt{D}}{2 a}},
$$

therefore, for $w_{0 j}(0)>\frac{b+\sqrt{D}}{2 a}$ is $C<0$ and for $w_{0 j}(0)<\frac{b-\sqrt{D}}{2 a}$ is $C>0$, from which it follows that, from the perspective of zero initial conditions, the value $w_{0 j}(0)$ is always well defined. But it always lies outside the interval $\left[\frac{b-\sqrt{D}}{2 a}, \frac{b+\sqrt{D}}{2 a}\right]$ and, 
therefore, it can never become zero, i.e., this solution of adaptive dynamics cannot meet the requirement of zero initial conditions.

Secondly, let us consider $w_{0 j} \in\left(\frac{b-\sqrt{D}}{2 a}, \frac{b+\sqrt{D}}{2 a}\right)$. Then, we get a sigmoidal behavior (see Figure 2)

i.e.,

$$
\mathrm{e}^{-\sqrt{D}(T-C)}=\frac{\frac{b+\sqrt{D}}{2 a}-w_{0 j}}{w_{0 j}-\frac{b-\sqrt{D}}{2 a}},
$$

$$
w_{0 j}(T)=\frac{1}{2 a}\left(b-\sqrt{D} \frac{\mathrm{e}^{-\sqrt{D}(T-C)}-1}{\mathrm{e}^{-\sqrt{D}(T-C)}+1}\right),
$$

i.e.,

$$
\lim _{T \rightarrow \infty} w_{0 j}(T)=w_{0 j}(\infty)=\frac{b+\sqrt{D}}{2 a}=I_{0}
$$

and

$$
\lim _{T \rightarrow-\infty} w_{0 j}(T)=w_{0 j}(-\infty)=\frac{b-\sqrt{D}}{2 a}=-\frac{I_{j}}{I_{0}}
$$
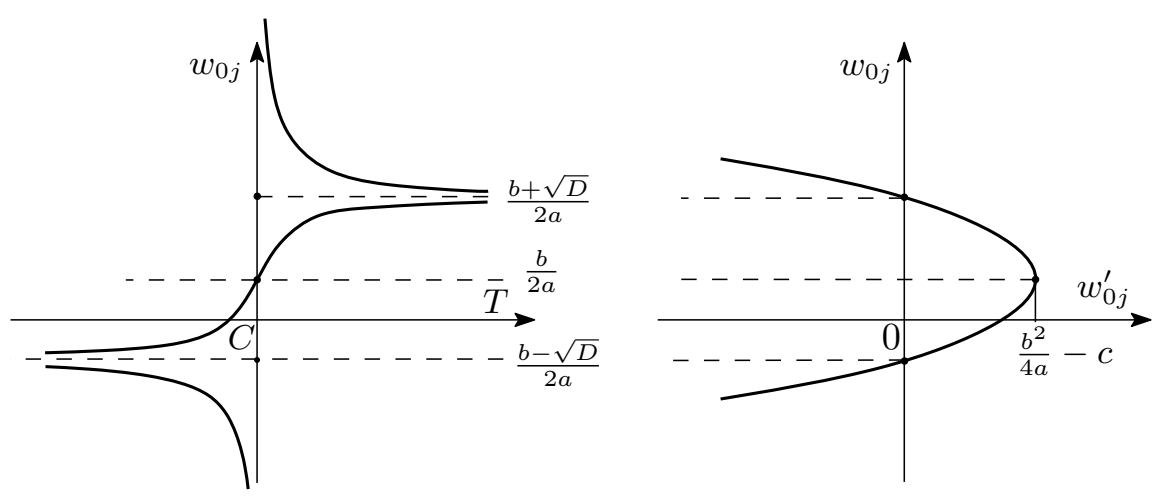

Figure 2. Dependency of synaptic weights on time or on its derivative.

For $T=0$ we get

i.e., for zero initial conditions,

$$
C=\frac{1}{\sqrt{D}} \ln \frac{\frac{b+\sqrt{D}}{2 a}-w_{0 j}(0)}{w_{0 j}(0)-\frac{b-\sqrt{D}}{2 a}},
$$

$$
C=\frac{1}{\sqrt{D}} \ln \frac{\sqrt{D}+b}{\sqrt{D}-b} .
$$

Therefore, only for $I_{0}^{2}=I_{j}$, we have $C=0$.

The general solution of adaptive dynamics (3.1) implies that, if the synaptic weights of learned network have been set to the desired values before starting the adaptation, i.e., on the limit values in infinite time of adaptive dynamics, adaptive 
dynamics does not make sense and, therefore, it does not make sense to learn the learned network.

Otherwise, the learned network function (2.3) for the reverse flow of time, a network remembering the future is obtained in the form

$$
\vec{F}\left(\left[I_{0}, 0,0,0\right]\right)=\left[I_{0},-I_{1},-I_{2},-I_{3}\right]
$$

and for the normal flow of time, a network remembering the past is obtained in form

$$
\vec{F}\left(\left[I_{0}, 0,0,0\right]\right)=\left[I_{0}, I_{0}^{2}, I_{0}^{2}, I_{0}^{2}\right] .
$$

Therefore, a network function is dependent on orientation of the perceptual arrow of time.

From the particular solution of adaptive dynamics (3.3) for the zero initial conditions and zero integration constant during the normal flow of time, we obtain the learned network function (2.3) in the form

$$
\vec{F}\left(\left[I_{0}, 0,0,0\right]\right)=\left[I_{0}, I_{1}, I_{2}, I_{3}\right] .
$$

\section{Outstar NETWORK}

Let us have the neural network (see Figure 3) where, for simplicity, we regard the activation and adaptation function as identity and we set the measure of plasticity or elasticity equal to one or zero. Then, assuming zero initial conditions for $-\vartheta_{j}=I_{j}>0$ where $j \in\{0,1,2,3\}$, we obtain from (2.1) and (2.2) the next active and adaptive dynamics.

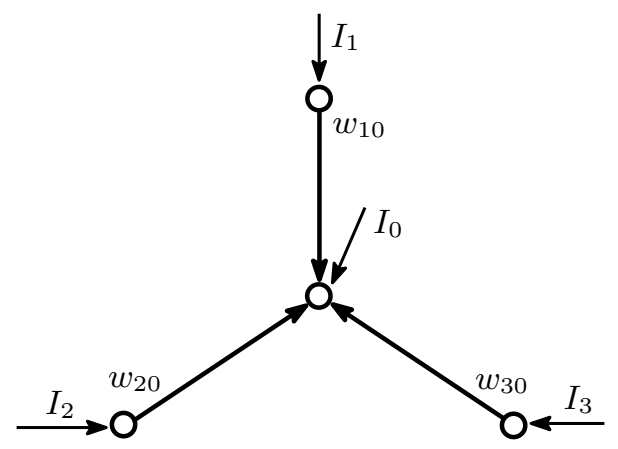

Figure 3. Outstar network.

Active dynamics:

$$
\frac{\mathrm{d}}{\mathrm{d} t} x_{0}(t)+x_{0}(t)=\sum_{j} x_{j}(t-\Delta t) w_{j 0}+I_{0},
$$

i.e.,

$$
x_{0}(t)=\sum_{j} I_{j} w_{j 0}\left(1-q(t) \mathrm{e}^{-t}\right)+I_{0}\left(1-\mathrm{e}^{-t}\right), \quad q(t)=\left(1+t \mathrm{e}^{\Delta t}\right),
$$


i.e.,

$$
\lim _{t \rightarrow \infty} x_{0}(t)=x_{0}(\infty)=\sum_{j} I_{j} w_{j 0}+I_{0}
$$

and for $j \in\{1,2,3\}$

$$
\frac{\mathrm{d}}{\mathrm{d} t} x_{j}(t)+x_{j}(t)=I_{j},
$$

i.e.,

$$
x_{j}(t)=I_{j}\left(1-\mathrm{e}^{-t}\right),
$$

i.e.,

$$
\lim _{t \rightarrow \infty} x_{j}(t)=x_{j}(\infty)=I_{j} .
$$

Adaptive dynamics:

$$
\frac{\mathrm{d}}{\mathrm{d} T} w_{j 0}(T)=x_{j}(\infty) x_{0}(\infty)
$$

i.e.,

$$
\frac{\mathrm{d}}{\mathrm{d} T} w_{j 0}(T)=I_{j}\left(\sum_{i} I_{i} w_{i 0}(T)+I_{0}\right)
$$

for $i, j \in\{1,2,3\}$, i.e., generally

$$
w_{j 0}(T)=w_{j 0}(0)+\frac{I_{j}}{\omega} C\left(\mathrm{e}^{\omega T}-1\right)
$$

where $\omega=|\vec{I}|^{2}$ for $\vec{I}=\left[I_{1}, I_{2}, I_{3}\right]$ and where integration constant

$$
C=\sum_{i} I_{i} w_{i 0}(0)+I_{0}
$$

i.e.,

$$
\lim _{T \rightarrow \infty} w_{j 0}(T)=w_{0 j}(\infty)=\infty
$$

From this it follows that there is no point in letting the adaptive dynamics time tend to infinity. Let the stimuli $I_{0}$ and $\vec{I}$ operate on a network during period of adaptation $\left(T_{0}, T_{1}\right)$ and, then, let the stimuli $J_{0}$ and $\vec{J}=\left[J_{1}, J_{2}, J_{3}\right]$ operate on a network during period of adaptation $\left(T_{1}, T_{2}\right)$, where

$$
T_{0}=0, \quad T_{1}=|\vec{I}|^{-2} \ln 2, \quad T_{2}=|\vec{J}|^{-2} \ln 2 .
$$

From the particular solution of adaptive dynamics (4.1) defined by zero initial condition for period of adaptation $\left(T_{0}, T_{1}\right)$ we obtain the network function $(2.3)$ in the form

$$
\vec{F}\left(\left[0, I_{1}, I_{2}, I_{3}\right]\right)=\left[I_{0}, I_{1}, I_{2}, I_{3}\right]
$$

because

$$
\sum_{j} I_{j} w_{j 0}\left(T_{1}\right)=I_{0}
$$


Next, from the particular solution of adaptive dynamics (4.1) defined by initial condition $w_{j 0}\left(T_{1}\right)=|\vec{I}|^{-2} I_{j} I_{0}$ for period of adaptation $\left(T_{1}, T_{2}\right)$, we obtain

$$
w_{j 0}\left(T_{2}\right)=\frac{I_{j} I_{0}}{|\vec{I}|^{2}}+\frac{J_{j} J_{0}}{|\vec{J}|^{2}}+\frac{(\vec{I} \cdot \vec{J})}{|\vec{I}|^{2}|\vec{J}|^{2}} J_{j} I_{0},
$$

i.e.,

$$
\begin{aligned}
& \sum_{j} I_{j} w_{j 0}\left(T_{2}\right)=I_{0}+\frac{(\vec{I} \cdot \vec{J})}{|\vec{J}|^{2}} J_{0}+\frac{(\vec{I} \cdot \vec{J})}{|\vec{I}|^{2}|\vec{J}|^{2}}(\vec{I} \cdot \vec{J}) I_{0}, \\
& \sum_{j} J_{j} w_{j 0}\left(T_{2}\right)=J_{0}+\frac{(\vec{I} \cdot \vec{J})}{|\vec{I}|^{2}} I_{0}+\frac{(\vec{I} \cdot \vec{J})}{|\vec{I}|^{2}|\vec{J}|^{2}}(\vec{J} \cdot \vec{J}) I_{0}
\end{aligned}
$$

i.e.,

$$
\begin{aligned}
& \sum_{j} I_{j} w_{j 0}\left(T_{2}\right)=I_{0}+\frac{|\vec{I}|}{|\vec{J}|} J_{0} \cos \phi(\vec{I}, \vec{J})+I_{0} \cos ^{2} \phi(\vec{I}, \vec{J}), \\
& \sum_{j} J_{j} w_{j 0}\left(T_{2}\right)=J_{0}+\frac{|\vec{J}|}{|\vec{I}|} I_{0} \cos \phi(\vec{I}, \vec{J})+\frac{|\vec{J}|}{|\vec{I}|} I_{0} \cos \phi(\vec{I}, \vec{J}) .
\end{aligned}
$$

Therefore, for mutually orthogonal inputs $\vec{I}, \vec{J}$ the network function (2.3) is obtained in the form

$$
\vec{F}\left(\left[0, I_{1}, I_{2}, I_{3}\right]\right)=\left[I_{0}, I_{1}, I_{2}, I_{3}\right], \quad \vec{F}\left(\left[0, J_{1}, J_{2}, J_{3}\right]\right)=\left[J_{0}, J_{1}, J_{2}, J_{3}\right] .
$$

\section{Conclusion}

During instar learning $(\beta=1)$, information signal $I_{0}$ (presynaptic activity) passing through synapsis over a long enough period is really stored in synaptic linkage memory, therefore the memory capacity is limited by the value $I_{0}$ (see Figure 4).

During outstar learning $(\beta=0)$, normalized information signal $I_{j}$ passing through synapsis over a given period and weighted by stimulus $I_{0}$ (postsynaptic activity) injected to a signal receiving neuron is stored in synaptic linkage memory. Analogously, we can indefinitely store into memory the values of pairs of stimuli $\vec{J}$ and $J_{0}$ injected into both layers of neurons, i.e., into chip and center of star respectively, therefore, the memory capacity is unlimited (see Figure 4).

The instar network function can be interpreted as a conditioned reflex model where, if the stimulus $I_{0}$ (sound perception) is in a certain proportion to the stimulus $I_{j}$ (odor perception) and the stimuli mentioned will be parallelly attached to the network over a long enough period for the network to be able to adapt to them, the sound without the odor causes an animal salivation (Pavlov's famous experiment).

The outstar network function can be interpreted as an associative memory, where with sufficient contrast (orthogonality) of stimuli $\vec{I}$ and $\vec{J}$ the memory during its gradual submission recalls its associations $I_{0}$ and $J_{0}$ respectively.

Instar network is the precedent of competition networks as a self-organizing map $[11,12]$ or counter-propagation network $[7,8]$ and outstar network is the precedent 


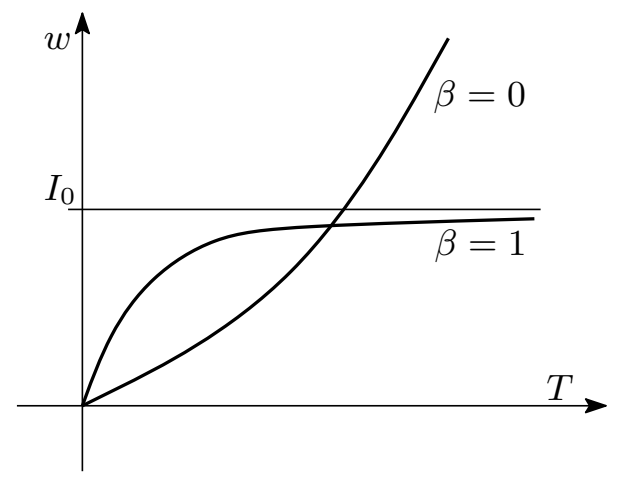

Figure 4. Comparison of instar and outstar learning.

of associative memories as Hopfield $[9,10]$ and bidirectional memory $[13,14]$ respectively with Hebbian learning rule [6].

\section{REFERENCES}

[1] G. A. Carpenter and S. Grossberg, A massively parallel architecture for a self-organizing neural pattern recognition machine, Comput. Vision Graph. 37 (1987), 54-115.

[2] G. A. Carpenter and S. Grossberg, ART 2: Self-organization of stable category recognition codes for analog input patterns, Appl. Optics 26 (1987), 4919-4930.

[3] G. A. Carpenter, S. Grossberg and D. B. Rosen, ART 2-A: An adaptive resonance algorithm for rapid category learning and recognition, Neural Networks 4 (1991), 493-504.

[4] S. Grossberg, Studies of Mind and Brain, Boston Studies in the Philosophy and of Science 70, Reidel, Dordrecht, 1982.

[5] S. Grossberg, The Adaptive Brain, North-Holland, 1987.

[6] D. O. Hebb, The Organization of Behavior: A Neuropsychological Theory, Wiley \& Sons, 1949.

[7] R. Hecht-Nielsen, Counterpropagation networks, Appl. Optics 26 (1987), 4979-4984.

[8] R. Hecht-Nielsen, Applications of counterpropagation networks, Neural Networks 1 (1988), 131-139.

[9] J. J. Hopfield, Neural networks and physical systems with emergent collective computational abilities, P. Natl. Acad. Sci. USA 79 (1982), 2554-2558.

[10] J. J. Hopfield, Artificial neural networks, IEEE Circuits Device. 4 (1988), 3-10.

[11] T. Kohonen, Self-organized formation of topologically correct feature maps, Biol. Cybern. 43 (1982), 59-69.

[12] T. Kohonen, The self-organizing map, P. IEEE 78 (1990), 1464-1480.

[13] B. Kosko, Adaptive bidirectional associative memories, Appl. Optics 26 (1987), 4947-4960.

[14] B. Kosko, Bidirectional associative memories, IEEE T. Syst. Man Cyb. 18 (1988), 49-60.

Miloš Křivan, Faculty of Informatics and Statistics of University of Economics in Prague e-mail: krivanm@vse.cz 
